\title{
Effect of a female labour companion and of educating her regarding support during labour on perinatal and labour outcomes
}

\author{
Senanayake $\mathrm{H} \mathrm{M}^{1}$, Somawardana U A B P ${ }^{2}$, Samarasinghe $\mathbf{M}^{2}$
}

\section{Abstract}

Objectives: To study the effect of the presence of a labour companion from the mother's social network and the effect of giving to the companion an education regarding support to the parturient.

Design: This was a comparative trial in a University Obstetric Unit. Sixty women were randomly distributed in two groups who were allowed female companions of their choice, one where the labour companion was given an education $(\mathrm{A})$ and a second where the companion was not given such an education (B). Group $C$ comprised of 30 women who did not have a companion as routinely practiced in our hospital. Perception of control over self during labour was assessed using the validated version of the Labour Agentry Scale (LAS-10) and perinatal events were recorded. Anova test and respective 95\% confidence interval were used in statistical analyses.

Results: Maternal satisfaction was significantly higher in the group A (Group A: mean $=56.10$, Group $B=48.40$, Group $C=45.93 ; p<0.05$ ) and the number of breastfeeds in the first 12 hours in group $B(A:$ mean $=5.8 ; S D=1.45 B: 6.03,1.09 ; C 5.0,1.2, p<0.05)$. There was a favourable trend in the need for augmentation of labour, with the following numbers not requiring such intervention: $A=10(33.3 \%) ; B=13(43.3 \%) ; C=9(30 \%)$. No difference was demonstrated in the use of analgesics, mode of delivery or presence of meconium staining of liquor.

Conclusions: A female labour companion of the mother's choice has a positive effect on the mother's experience of labour and perinatal events, irrespective of the companion receiving an education.

\section{Introduction}

Continuous nonmedical support during labour has been shown to produce favorable intrapartum, perinatal and neonatal outcomes ${ }^{1}$. A systematic review of 15 randomized controlled trials on continuous support during labor conducted under diverse settings and involving

${ }^{1}$ Professor in Obstetrics and Gynaecology and Head of Department,

${ }^{2}$ Research Assistants, Department of Obstetrics and Gynaecology, Faculty of Medicine, University of Colombo, Sri Lanka.

Correspondence: Hemantha M. Senanayake

E-mail: senanayakeh@gmail.com

Competing interests: None.
12,791 women provides robust evidence of these benefits ${ }^{2}$. This showed that women who received continuous intrapartum support were less likely to require intrapartum analgesia or an operative birth and that they were less likely to deliver a baby with 5 minute Apgar scores less than 7 or to report dissatisfaction with their childbirth experiences ${ }^{2}$. The review concluded that all women should have continuous support during labour. Continuous support from a person who is present solely to provide support, not a member of the woman's social network, experienced in providing labour support and has at least a modest amount of training, appeared to be most beneficial ${ }^{2}$. A person who provides nonmedical emotional, physical and informational support to a woman during and after labour is referred to as a doula. By definition, this term would include members of the woman's family and social network. A review of evidence-based labour and delivery management rated support by a doula in labour as a Grade A recommendation and described it as one of the most effective interventions in labour ${ }^{3}$. A few countries offer training courses for women who want to be doulas. In developed countries, the norm is for labouring women to be supported by their male partners and others of their choice. However, the outcome of support during labour by a male partner remains controversial ${ }^{4,5}$.

In most low-resource settings such as Sri Lanka however, the infrastructure in the majority of labour wards would not be able to support the presence of a male partner. In contrast, minor administrative interventions would make the presence of a female companion during labour eminently feasible. Under the current policies followed in Sri Lankan government hospitals, women labour for hours without contact with family and close associates. In a time of great emotional demand, this will be an additional burden on the ability of the mother to cope. In this context, it is most likely that companionship during labour could influence at least the emotional experience of labour.

There have been only a few studies that have assessed the effects of support by a family member or friend and these suggest that such support may not always be a positive experience $^{6}$. We chose this aspect since it seems the most practicable way in which support could be provided to labouring mothers in Sri Lanka and in other developing countries. Hodnett's review ${ }^{2}$ showed that the results of support given by women from within the mother's social or family network were inferior to those produced by 
those from outside. It is possible however, that Sri Lankan female companions, given their high health awareness and literacy could produce different results. If it is found to be useful, support by a female companion could be implemented as a useful intervention in low resource settings. This may prove to be one that is more feasible than provision of a doula from outside a woman's social network or to allow the presence of her male partner.

Aims of the present study were to evaluate the effect of support during labour given by a female companion from the mother's social network and to evaluate what effect giving an education to the labour companion would have on outcomes of labour.

\section{Method}

We conducted a comparative trial in the Professorial Unit of the De Soysa Hospital for Women, Colombo, Sri Lanka from 1st September 2008 to 1st March 2009. Ethical aspects of the study were reviewed by the Ethics Review Committee of the Faculty of Medicine, University of Colombo.

Sixty women were recruited from the antenatal clinics and randomly allocated to two groups: A and B, who were to be allowed to have a female companion of their choice throughout labour. The nominated labour companions of Group A were educated regarding labour and about ways of providing support to the labouring woman according to a predetermined format. They were educated regarding issues given in Table 1 in simple language. These sessions were conducted as one-to-one sessions.

The companions of Group B were not given any formal instructions on how to support the labouring mother, but were told about the basic rules to follow in the labour ward to minimize interference with ward procedures. They were free to support the mother within these instructions.

Table 1 shows the points discussed with the companions of Group A to educate them regarding support during labour.

\section{Table 1. Areas on which the companions of Group} A were educated regarding labour

01. Stages, normal length, symptoms and signs of labour

02. Types of analgesia and other medications available for use during labour

03. How to support the mother and how to deal with her during labour i.e. the less talk the better, avoid eye contact, allow and encourage freedom of movement

04. Special considerations in addressing the mother:

a. Be quiet and confidential, speak softly

b. Act more like a partner and not like a professional

C. Do not hold any authority over the mother at any point

05. Remind the mother to go to the toilet regularly during labour (depending on circumstances) and to keep the bladder empty

06. Physical comfort of the mother:

a. Keep her cool. Use cold towels to wipe the face. Offer liquids according to unit policy

b. Massage softly: stroking the back in early first stage while sitting on the bed and sacral massage while lying in left lateral position

07. Don't acknowledge labour as a painful process but perceive it as a sacred process of giving birth to another human being

08. Synchronize breathing to avoid hyperventilation by the mother

The eligibility criteria for recruitment were primigravidae between 28-36 weeks of period of gestation, uneventful singleton pregnancy, cephalic presentation, availability of a female companion of her choice to be present during the process of labour and their ability to read Sinhala or Tamil or English. They were provided with an information sheet at that stage to make an informed decision with the rest of the family. Multipara, those with multiple pregnancy, abnormal presentation, prematurity or postmaturity, maternal medical or other complications, those with an indication for elective caesarean section, fetal malformations and those who did not consent were excluded.

The companions were allowed to remain in the labour ward throughout labour but were told they may be asked to leave the ward in case the staff deemed it necessary in case of an emergency. Permission was obtained from the Director and Matron of the Hospital to allow labour companions. We conducted awareness sessions for the ward staff on companionship in labour since the concept was a new and challenging one for them.

The first primigravidae who had a normal delivery after a woman from Groups A or B was recruited to Group $C$ as a control until 30 women were recruited. The routine practice in our hospital at the time was for women not to be allowed a companion.

Perception of control over self during labour was assessed using a validated version of the Labour Agentry Scale 7,8 (LAS-10). This has been the tool used for this purpose in all studies where it has been necessary to study this aspect. The Sinhala and Tamil versions of the scale were validated by seeking opinions of 10 experts. The questionnaire was administered to mothers within 24 hours of the birth. Perinatal events and the number of breast feeds in the first 24 hours were recorded by direct inquiry and by perusing the clinical records. Anova test and respective $95 \%$ confidence interval were used in statistical analyses. 
Table 2. Comparison between the three groups with regard to the perceived satisfaction with labour and perinatal events

\begin{tabular}{lllll}
\hline Criterion & Group A & Group B & Group C & $p$ value \\
\hline Perceived satisfaction with labour & $56.10(9.02)$ & $48.40,7.36$ & $45.93,9.29$ & $<0.05$ \\
Breast feeds in first 12 hours & $5.8(1.45)$ & $6.03(1.09)$ & $5.0(1.20)$ & $<0.05$ \\
Number needing augmentation of labour & $20(66.6 \%)$ & $17(56.7 \%)$ & $21(70 \%)$ & $\mathrm{NS}$ \\
Length of active labour (hours) *excludes & $5.41(2.54)$ & $6.12(2.65)$ & $7.02(3.21)$ & $\mathrm{NS}$ \\
those undergoing caesarean section & $24(80 \%)$ & $23(76 \%)$ & $23(76 \%)$ & $\mathrm{NS}$ \\
Normal delivery & $5(16 \%)$ & $5(16 \%)$ & $4(13.3 \%)$ & 3 \\
Emergency cesarean section & 1 & 2 & - & 02 \\
Assisted vaginal delivery & - & & - \\
Apgar score $\leq 7$ & & &
\end{tabular}

\section{Results}

From the total of 60 women in Groups A and B, 44 (73.33\%) selected their mother as the support person and the rest, another female relative. There was no significant difference in the mean age of the 3 study groups $(A=24.17$ years, $B=24.40, C=24.27)$.

Comparison between the three groups with regard to the perceived satisfactions with labour and perinatal events is given in Table 2 .

There was no statistical difference between the groups with respect to the type of analgesic used, the frequency of administration of analgesics, mode of delivery or presence of meconium staining of liquor.

\section{Discussion}

Before childbirth moved from the home to hospital, it was common practice to have female family members and close friends attending on labouring mothers. The findings of this study confirm the value of this practice. Maternal satisfaction in the groups that had a labour companion was significantly higher, as reflected by high LAS-10 scores. The score was highest in the group who had a companion who underwent education regarding support during labour.
Hodnett's review ${ }^{2}$ showed that women most likely to benefit were likely to be those who received support from women who had at least a minimal education on it. In this study, the beneficial effects such as the number of feeds in the first 12 hours were seen even in the group of women whose companions had not received formal instruction. However, we would describe the education the companions of group A were given as very basic and easily imparted even in the setting of a busy labor ward.

The significantly higher number of breastfeeds in the first 24 hours in the supported groups is in concordance with results of previous studies ${ }^{9-11}$. This indicates that companionship in labour could have a major impact on establishment of breastfeeding. For women in developing countries and their healthcare systems, this could prove to be a substantial advantage.

Two babies in the group that did not have a companion were born with Apgar scores below 7, whereas none in the two groups that had a companion had low scores. Although this was not statistically significant and not an aspect we studied specifically, we suspect that this difference was because women with companions received closer monitoring. This could have been an indirect result of the presence of a companion.
This study was aimed at reproducing favourable results of previous studies evaluating the presence of a female during labour companion in a low-resource setting. It has shown beneficial effects of the mere presence of a female labour. Many of the favourable perinatal outcomes were seen irrespective of the companion receiving an education on supporting the parturient. There was also a clear trend towards shorter labours in the groups that had a companion.

Apart from the scientific aspects, a companion will also empower women indirectly and improve the way care providers interact with them. In addition to being an effective, low cost intervention in labour, support by a companion could also contribute to improving the overall quality of maternity care in Sri Lanka. During the period of this study we found it could be implemented easily and the attitude of the staff towards it was overwhelmingly positive. We recommend that Sri Lankan hospitals adopt allowing a female companion of the labouring mother's choice as policy.

\section{Acknowledgments}

The Labour Agentry Scale (LAS-10) was used with the kind permission of Professor Ellen Hodnett, Professor and Heather M Reisman, Chair in 
Perinatal Nursing Research, University of Toronto. The authors wish to acknowledge the advice of Dr. Carukshi Arambepola, Senior Lecturer, Department of Community Medicine, Faculty of Medicine, University of Colombo, The Director, the doctors and the entire nursing and midwifery staff of ward 15 of the De Soysa Hospital for Women (DMH), Colombo 08. Mrs. Mohanie Ahangama, Prenatal Educator advised on educating Group A on support during labour.

\section{REFERENCES}

1. Madi BC, Sandall J, Bennett R, MacLeod C. Effects of female relative support in labor: a randomized controlled trial. Birth. 1999 Mar; 26(1): 4-8.

2. Hodnett ED, Gates D, Hofmeyr GJ, Sakala C. Continuous support for women during childbirth. The Cochrane Database of Systemic Reviews 2003, Issue 3. Art. No. : CD003766.DOI.10.1002/14651858. CD003766.

3. Berghella V, Baxter JK, Chauhan SP.
Evidence-based labour and delivery management. Am j Obstet Gynaecol. 2008 Nov; 199(5): 445-54.

4. Susan K. McGrath PhD, John H. Kennell MD. A Randomized Controlled Trial of Continuous Labor Support for Middle-Class Couples: Effect on Cesarean Delivery Rates. Birth. 2008 June; 35(2): 97-8.

5. Bertsch TD, Nagashima-Whalcn L, Dykcman S, Kennell JH, McGrath S. Labor support by first-time fathers: direct observations with a comparison to experienced doulas. Journal of Psychosomatic Obstetrics \& Gynecology, Dec 1990: 11(4), 251-60.

6. Keirse M, Enkin M, Lumley J. Social and professional support during child birth. Effective care in pregnancy and childbirth, 1989; 2: 805-14.

7. Hodnett ED, Simmons-Tropea DA (1987) The Labour Agentry Scale: Psychometric Properties of An Instrument Measuring Control During Childbirth. Research in Nursing and Health. 10: 301-10.

8. Hodnett E. Labour agentry scale: A measure of a woman's sense of control over the childbirth experience; your feelings during labour. In: Redman, Barbara K, editors. Measurement tools in patient education. New York: Springer Pub, Co, 1983: 276-8.

9. Hofmeyr GJ, Nikodem VC, Wolman WL et al. Companionship to modify the clinical birth environment: Effects on progress of labour and breast feeding. $\mathrm{Br} \mathrm{J}$ Obstet Gynaecol. 1991; 9(8): 756-64.

10. Bello MIO, Adedokun BO, Ojengbede OA. Social support during childbirth as a catalyst for early breast feeding initiation for first time Nigerian mothers. International breast feeding journal. 2009 Dec [cited 2009 Dec 10]; 4(16): http://www.international breastfeedingjournal.com/content/4/1/16. Accessed 30 Oct 2011.

11. Langer A, Campero L, Garia C, Reynoso S. Effects of psychosocial support during labour and childbirth on breast feeding, medical interventions, and mothers' wellbeing in a Mexican public hospital: a randomized clinical trial. Br J Obstet Gynaecol. 1998; (105): 1056-63.

12. Campbell D, Scott KD, Kalus MH, Falk M. Female relatives or friends trained as labour doulas: Outcome at 6 to 8 weeks postpartum. Birth. 2007 Sept; 34(3): 220-27. 